\title{
Three-dimensional envelope instability in periodic focusing channels
}

\author{
Ji Qiang* \\ Lawrence Berkeley National Laboratory, Berkeley, California 94720, USA
}

(Received 9 December 2017; published 20 March 2018)

\begin{abstract}
The space-charge driven envelope instability can be of great danger in high intensity accelerators and was studied using a two-dimensional (2D) envelope model and three-dimensional (3D) macroparticle simulations before. In this paper, we study the instability for a bunched beam using a three-dimensional envelope model in a periodic solenoid and radio-frequency (rf) focusing channel and a periodic quadrupole and rf focusing channel. This study shows that when the transverse zero current phase advance is below $90^{\circ}$, the beam envelope can still become unstable if the longitudinal zero current phase advance is beyond $90^{\circ}$. For the transverse zero current phase advance beyond $90^{\circ}$, the instability stopband width becomes larger with the increase of the longitudinal focusing strength and even shows different structure from the 2D case when the longitudinal zero current phase advance is beyond $90^{\circ}$. Breaking the symmetry of two longitudinal focusing rf cavities and the symmetry between the horizontal focusing and the vertical focusing in the transverse plane in the periodic quadrupole and rf channel makes the instability stopband broader. This suggests that a more symmetric accelerator lattice design might help reduce the range of the envelope instability in parameter space.
\end{abstract}

DOI: 10.1103/PhysRevAccelBeams.21.034201

\section{INTRODUCTION}

The envelope instability as a space-charge driven collective instability presents a potentially great danger in high intensity accelerators by causing beam size blow up and quality degradation. It has been studied theoretically [1-8] and experimentally [9-11] since the 1980s. In recent years, there was growing interest in further understanding this instability and other structural resonances [12-22]. Some of those studies were summarized in a recently published monograph [23]. However, most of those theoretical studies were based on a two-dimensional model. Three-dimensional macroparticle simulations were carried out for a bunched beam under the guidance of the two-dimensional envelope instability model [16,19]. It was found in Ref. [16] that the instability stopband from the 3D macroparticle simulation is broader than that from the 2D envelope model. Furthermore, the effect of the longitudinal synchrotron motion has not been systematically studied in those macroparticle simulations and is missed in the $2 \mathrm{D}$ envelope instability model. In this paper, we studied the envelope instability for a bunched beam using a set of three-dimensional envelope equations in periodic focusing channels. This analysis can be

\footnotetext{
*jqiang@lbl.gov
}

Published by the American Physical Society under the terms of the Creative Commons Attribution 4.0 International license. Further distribution of this work must maintain attribution to the author(s) and the published article's title, journal citation, and DOI. used to systematically study the effect of longitudinal synchrotron motion on the instability stopband for a bunched beam. It can also be used to explore the stability in a fully 3D parameter space and to provide guidance for $3 \mathrm{D}$ macroparticle simulations.

The organization of this paper is as follows: after the introduction, we review the 2D envelope instability model in Sec. II; we present the 3D envelope instability model in Sec. III; we present numerical study of the envelope instability in a periodic transverse solenoid and longitudinal rf focusing channel in Sec. IV; I present numerical study of the envelope instability in a periodic transverse quadrupole and longitudinal rf focusing channel in Sec. V; and draw conclusions in Sec. VI.

\section{TWO-DIMENSIONAL ENVELOPE INSTABILITY MODEL}

For a two-dimensional coasting beam subject to external periodic focusing forces and linear space-charge forces, the two-dimensional envelope equations for the transverse rms sizes as functions of the distance $s$ along the axis of the accelerator are given as [2,24]:

$$
\begin{aligned}
& \frac{d^{2} X}{d s^{2}}+k_{x}^{2}(s) X-\frac{K / 2}{X+Y}-\frac{\epsilon_{x}^{2}}{X^{3}}=0 \\
& \frac{d^{2} Y}{d s^{2}}+k_{y}^{2}(s) Y-\frac{K / 2}{X+Y}-\frac{\epsilon_{y}^{2}}{Y^{3}}=0
\end{aligned}
$$

where $X$ and $Y$ are horizontal and vertical rms beam sizes respectively, $k_{x}^{2}$ and $k_{y}^{2}$ represent the external periodic 
focusing forces $\left(k_{x}(s)=k_{y}(s)=q B(s) /\left(2 p_{0}\right)\right.$ for solenoids and $k_{x, y}^{2}(s)= \pm q G(s) / p_{0}$ for quadrupoles, where $B$ is the solenoid root mean-squared magnetic field along the axis and $G$ is the quadrupole gradient), $\epsilon_{x}$ and $\epsilon_{y}$ denote unnormalized rms emittances, and $K$ is the generalized perveance associated with the space-charge strength given by:

$$
K=\frac{q I}{2 \pi \epsilon_{0} p_{0} v_{0}^{2} \gamma_{0}^{2}}
$$

where $I$ is the current of the beam, $q$ is the charge of the particle, $\epsilon_{0}$ is the vacuum permittivity, $p_{0}$ is the momentum of the reference particle, $v_{0}$ is the speed of the reference particle, and $\gamma_{0}$ is the relativistic factor of the reference particle.

The above equations can be linearized with respect to a periodic solution (i.e. matched solution) as:

$$
\begin{aligned}
& X(s)=X_{0}(s)+x(s) \\
& Y(s)=Y_{0}(s)+y(s)
\end{aligned}
$$

where $X_{0}$ and $Y_{0}$ denote the periodic matched envelope solutions $\left[X_{0}(s)=X_{0}(s+L), X_{0}^{\prime}(s)=X_{0}^{\prime}(s+L), Y_{0}(s)=\right.$ $Y_{0}(s+L)$, and $\left.Y_{0}^{\prime}(s)=Y_{0}^{\prime}(s+L)\right]$ that can be found through the Newton search algorithm with a contraction map [25], and $x$ and $y$ denote small perturbations

$$
x(s) \ll X_{0}(s), \quad y(s) \ll Y_{0}(s)
$$

The match solutions are available as long as the zero current phase advances are below $180^{\circ}$.

The equations of motion for the small perturbations are given by:

$$
\begin{aligned}
& \frac{d^{2} x(s)}{d s^{2}}+a_{1}(s) x(s)+a_{12}(s) y(s)=0 \\
& \frac{d^{2} y(s)}{d s^{2}}+a_{2}(s) y(s)+a_{12}(s) x(s)=0
\end{aligned}
$$

where

$$
\begin{gathered}
a_{12}(s)=2 K /\left(X_{0}(s)+Y_{0}(s)\right)^{2} \\
a_{1}(s)=k_{x}^{2}(s)+3 \epsilon_{x}^{2} / X_{0}^{4}(s)+a_{12}(s) \\
a_{2}(s)=k_{y}^{2}(s)+3 \epsilon_{y}^{2} / Y_{0}^{4}(s)+a_{12}(s)
\end{gathered}
$$

With $\xi=\left(x, x^{\prime}, y, y^{\prime}\right)^{T}$, the prime denotes derivative with respect to $s$, and $T$ denotes the transpose of a matrix, the above equations can be rewritten in matrix notation as:

$$
\frac{d \xi}{d s}=A_{4}(s) \xi(s)
$$

with the periodic matrix

$$
A_{4}(s)=\left(\begin{array}{cccc}
0 & 1 & 0 & 0 \\
-a_{1}(s) & 0 & -a_{12}(s) & 0 \\
0 & 0 & 0 & 1 \\
-a_{12}(s) & 0 & -a_{2}(s) & 0
\end{array}\right)
$$

Let $\xi(s)=M_{4}(s) \xi(0)$ be the solution of above equation, substituting this equation into Eq. (12) results in

$$
\frac{d M_{4}(s)}{d s}=A_{4}(s) M_{4}(s)
$$

where $M_{4}(s)$ denotes the $4 \times 4$ transfer matrix solution of $\xi(s)$ and $M_{4}(0)$ is a $4 \times 4$ unit matrix. The matrix $A_{4}(s)$ is a periodic function of $s$ with a length of period $L$. Following the Floquet's theorem, the solution of $M_{4}(s)$ after $n$ lattice periods can be written as

$$
M_{4}(s+n L)=M_{4}(s) M_{4}(L)^{n}
$$

This matrix solution will remain finite as $n->\infty$, only if all amplitudes of the eigenvalues of the matrix $M_{4}(L)$ be less than or equal to one. Since the matrix $M_{4}(L)$ is a real symplectic matrix, the eigenvalues of the matrix occur both as reciprocal and as complex-conjugate pairs. Therefore, for stable solutions, all eigenvalues of the matrix $M_{4}(L)$ have to lie on a unit circle in the complex plane. The eigenvalues of the matrix $M_{4}(L)$ can be expressed in polar coordinates as:

$$
\lambda=|\lambda| \exp (i \phi)
$$

where the amplitude $|\lambda|$ of the eigenvalue gives the growth rate (or damping rate) of the envelope eigenmode through one lattice period and the phase shift $\phi$ of the eigenvalue gives the phase of the envelope mode oscillation through one period. For an unstable envelope mode, there are two possibilities [2]: (1) one or both eigenvalue pairs lie on the real axis: $\phi_{1,2}=180^{\circ}$, (2) the phase shift angles are equal: $\phi_{1}=\phi_{2}$. The first case can be seen as a half-integer parametric resonance between the focusing lattice and the envelope oscillation mode. The second case is a confluent resonance between two envelope oscillation modes since they have the same oscillation frequencies.

\section{THREE-DIMENSIONAL ENVELOPE INSTABILITY MODEL}

The 3D envelope equations have been used to study the halo particle formation mechanism (e.g., particle-core 
model) for a bunched beam in high intensity accelerators [26-29]. There, the mismatched envelope oscillation resonates with a test particle and drives the particle into large amplitude becoming a halo particle. The mismatched envelope oscillation itself is stable in that case. In this paper, I study the stability/instability of the mismatched envelope oscillation itself in periodic focusing channels.

For a 3D uniform density ellipsoidal beam inside a periodic focusing channel without acceleration, the threedimensional envelope equations are given as $[25,30]$ :

$$
\begin{gathered}
\frac{d^{2} X}{d s^{2}}+k_{x}^{2}(s) X-I_{x}(X, Y, Z) X-\frac{\epsilon_{x}^{2}}{X^{3}}=0 \\
\frac{d^{2} Y}{d s^{2}}+k_{y}^{2}(s) Y-I_{y}(X, Y, Z) Y-\frac{\epsilon_{y}^{2}}{Y^{3}}=0 \\
\frac{d^{2} Z}{d s^{2}}+k_{z}^{2}(s) Z-I_{z}(X, Y, Z) Z-\frac{\left(\epsilon_{z} / \gamma^{2}\right)^{2}}{Z^{3}}=0
\end{gathered}
$$

with

$I_{i}(X, Y, Z)=C \int_{0}^{\infty} \frac{d t}{\left(e_{i}^{2}+t\right) \sqrt{\left(X^{2}+t\right)\left(Y^{2}+t\right)\left(\gamma^{2} Z^{2}+t\right)}}$

where $X, Y$, and $Z$ are horizontal, vertical, and longitudinal rms beam sizes respectively, $k_{x}^{2}, k_{y}^{2}, k_{z}^{2}$ represent the external periodic focusing forces $\left(k_{z}^{2}=\omega q E_{0} T \sin \left(-\phi_{s}\right) /\left(m c^{2} \beta^{3} \gamma^{3}\right)\right.$ for longitudinal rf focusing [31]), $\epsilon_{x}, \epsilon_{y}$, and $\epsilon_{z}$ are unnormalized rms emittances, $e_{i}=X, Y, \gamma Z$, for $i=x, y, z$, and $C=\frac{1}{2} \frac{3}{4 \pi \epsilon_{0}} \frac{q}{m c^{2}} \frac{I}{f_{r f} \beta^{2} \gamma^{2}} \frac{1}{5 \sqrt{5}}$. Here, $\epsilon_{0}$ is the vacuum permittivity, $q$ the charge, $m c^{2}$ the rest energy of the particle, $c$ the light speed in vacuum, $I$ the average beam current, $f_{r f}$ the rf bunch frequency, $\beta=v / c, v$ the bunch velocity, and the relativistic factor $\gamma=1 / \sqrt{1-\beta^{2}}$. The nonlinear space-charge defocusing terms $I_{x, y, z}$ depend on the horizontal, vertical, and longitudinal rms beam sizes and provide coupling between the transverse and longitudinal envelope oscillations. The above 3D envelope equations can be derived from the definition of rms size and equations of motion in each dimension as shown in [30]. A detailed derivation of those equations including the 3D space-charge fields for a bunched beam with the ellipsoidal symmetry $(\rho(x, y, z ; s)=$ $\left.\rho\left(x^{2} / a^{2}+y^{2} / b^{2}+z^{2} / c^{2} ; s\right)\right)$ is given in the Appendix of Ref. [32]. It was pointed out in Ref. [30] that the space-charge form factor $1 / 5 \sqrt{5}$ for a uniform distribution depends only weakly on the type of distributions and is $1.01 / 5 \sqrt{5}$ for a parabolic distribution and $1.05 / 5 \sqrt{5}$ for a Gaussian distribution. The external periodic focusing forces $k_{x, y, z}(s)=$ $k_{x, y, z}(s+L)$ in above equations vary for different accelerator beam line elements. This envelope model is valid for a periodic accelerator transport channel no matter the external transverse focusing elements in a single period having a focusing drift defocusing drift (FODO) or a focusing drift focusing drift defocusing drift defocusing drift (FOFODODO) structure.

The above equations can be linearized with respect to periodic solutions (i.e. matched solutions) as:

$$
\begin{aligned}
& X(s)=X_{0}(s)+x(s) \\
& Y(s)=Y_{0}(s)+y(s) \\
& Z(s)=Z_{0}(s)+z(s)
\end{aligned}
$$

where $X_{0}, Y_{0}$, and $Z_{0}$ denote the periodic matched envelope solutions and $x, y$, and $z$ denote small perturbations

$$
x(s) \ll X_{0}(s), \quad y(s) \ll Y_{0}(s), \quad z(s) \ll Z_{0}(s) .
$$

The equations of motion for these small perturbations are given by:

$$
\begin{gathered}
\frac{d^{2} x}{d s^{2}}+a_{1}(s) x(s)+a_{12}(s) y(s)+\gamma^{2} a_{13}(s) z(s)=0 \\
\frac{d^{2} y}{d s^{2}}+a_{12}(s) x(s)+a_{2}(s) y(s)+\gamma^{2} a_{23}(s) z(s)=0 \\
\frac{d^{2} z}{d s^{2}}+a_{13}(s) x(s)+a_{23}(s) y(s)+a_{3}(s) z(s)=0
\end{gathered}
$$

where

$$
\begin{gathered}
a_{1}(s)=k_{x}^{2}+3 \epsilon_{x}^{2} / X_{0}^{4}-I_{x}\left(X_{0}, Y_{0}, Z_{0}\right)+3 X_{0}^{2} F_{x x} \\
a_{12}(s)=X_{0} Y_{0} F_{x y} \\
a_{13}(s)=X_{0} Z_{0} F_{x z} \\
a_{2}(s)=k_{y}^{2}+3 \epsilon_{y}^{2} / Y_{0}^{4}-I_{y}\left(X_{0}, Y_{0}, Z_{0}\right)+3 Y_{0}^{2} F_{y y} \\
a_{23}(s)=Y_{0} Z_{0} F_{y z}
\end{gathered}
$$

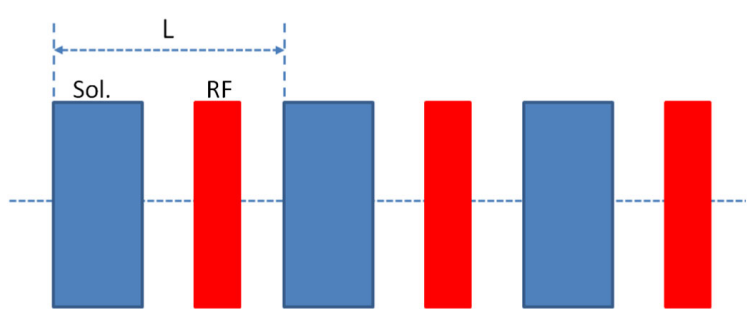

FIG. 1. Schematic plot of a periodic solenoid and rf channel. 

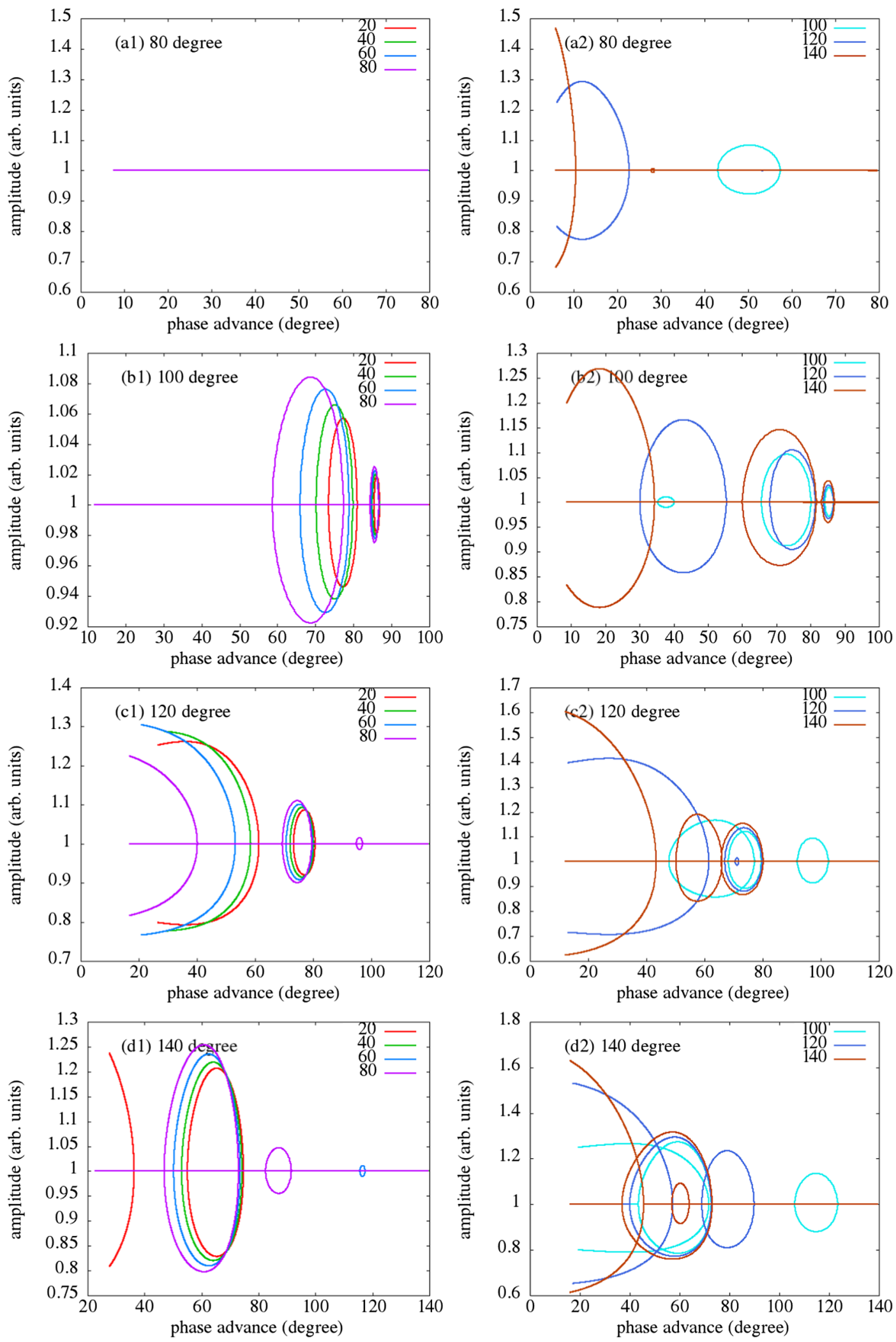

FIG. 2. The 3D envelope mode growth rate amplitudes as a function of depressed transverse phase advance with $20^{\circ}, 40^{\circ}, 60^{\circ}, 80^{\circ}$, $100^{\circ}, 120^{\circ}$, and $140^{\circ}$ zero current longitudinal phase advances for (a) $80^{\circ}$, (b) $100^{\circ}$, (c) $120^{\circ}$, and (d) $140^{\circ}$ zero current transverse phase advances in a periodic solenoid-rf channel. 

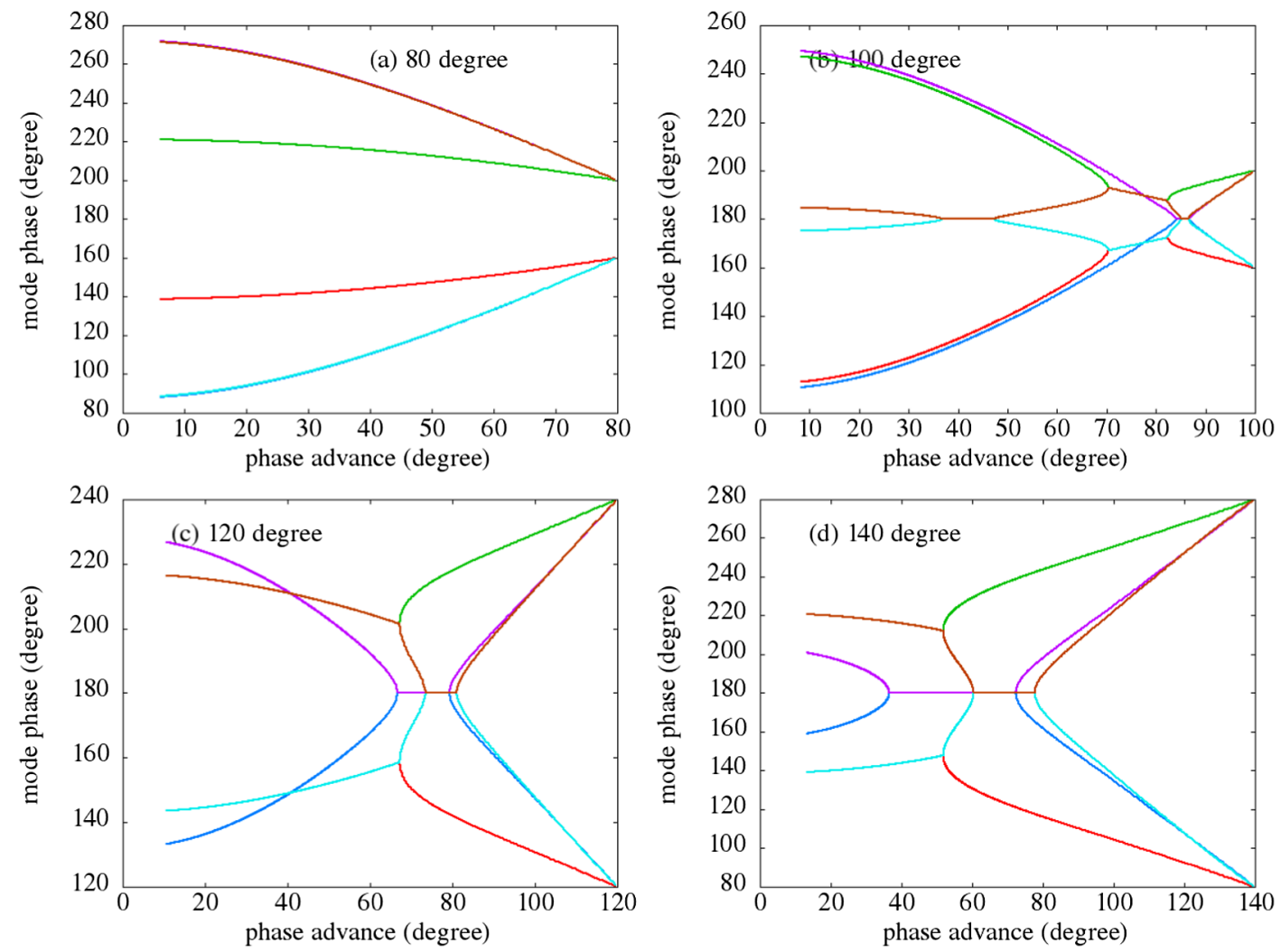

FIG. 3. The $3 \mathrm{D}$ envelope mode phases as a function of depressed transverse phase advance with (a) $80^{\circ}$, (b) $100^{\circ}$, (c) $120^{\circ}$, and (d) $140^{\circ}$ zero current longitudinal and transverse phase advances in a periodic solenoid-rf channel. The six colors are the phases of the six eigenvalues.

$a_{3}(s)=k_{z}^{2}+3\left(\epsilon_{z} / \gamma^{2}\right)^{2} / Z_{0}^{4}-I_{z}\left(X_{0}, Y_{0}, Z_{0}\right)+3 \gamma^{2} Z_{0}^{2} F_{z z} \quad F_{z z}=C \int_{0}^{\infty}\left(X_{0}^{2}+t\right)^{-1 / 2}\left(Y_{0}^{2}+t\right)^{-1 / 2}\left(Z_{0}^{2} \gamma^{2}+t\right)^{-5 / 2} d t$.

where

$F_{x x}=C \int_{0}^{\infty}\left(X_{0}^{2}+t\right)^{-5 / 2}\left(Y_{0}^{2}+t\right)^{-1 / 2}\left(Z_{0}^{2} \gamma^{2}+t\right)^{-1 / 2} d t$

$F_{x y}=C \int_{0}^{\infty}\left(X_{0}^{2}+t\right)^{-3 / 2}\left(Y_{0}^{2}+t\right)^{-3 / 2}\left(Z_{0}^{2} \gamma^{2}+t\right)^{-1 / 2} d t$

$F_{x z}=C \int_{0}^{\infty}\left(X_{0}^{2}+t\right)^{-3 / 2}\left(Y_{0}^{2}+t\right)^{-1 / 2}\left(Z_{0}^{2} \gamma^{2}+t\right)^{-3 / 2} d t$

$F_{y y}=C \int_{0}^{\infty}\left(X_{0}^{2}+t\right)^{-1 / 2}\left(Y_{0}^{2}+t\right)^{-5 / 2}\left(Z_{0}^{2} \gamma^{2}+t\right)^{-1 / 2} d t$

$F_{y z}=C \int_{0}^{\infty}\left(X_{0}^{2}+t\right)^{-1 / 2}\left(Y_{0}^{2}+t\right)^{-3 / 2}\left(Z_{0}^{2} \gamma^{2}+t\right)^{-3 / 2} d t$

With $\xi=\left(x, x^{\prime}, y, y^{\prime}, z, z^{\prime}\right)^{T}$, the above equations can be rewritten in matrix notation as:

$$
\frac{d \xi}{d s}=A_{6}(s) \xi(s)
$$

with the periodic matrix

$$
A_{6}(s)=\left(\begin{array}{cccccc}
0 & 1 & 0 & 0 & 0 & 0 \\
-a_{1}(s) & 0 & -a_{12}(s) & 0 & -\gamma^{2} a_{13}(s) & 0 \\
0 & 0 & 0 & 1 & 0 & 0 \\
-a_{12}(s) & 0 & -a_{2}(s) & 0 & -\gamma^{2} a_{23}(s) & 0 \\
0 & 0 & 0 & 0 & 0 & 1 \\
-a_{13}(s) & 0 & -a_{23}(s) & 0 & -a_{3}(s) & 0
\end{array}\right)
$$

Let $\xi(s)=M_{6}(s) \xi(0)$, substituting this equation into Eq. (40) results in

$$
\frac{d M_{6}(s)}{d s}=A_{6}(s) M_{6}(s)
$$



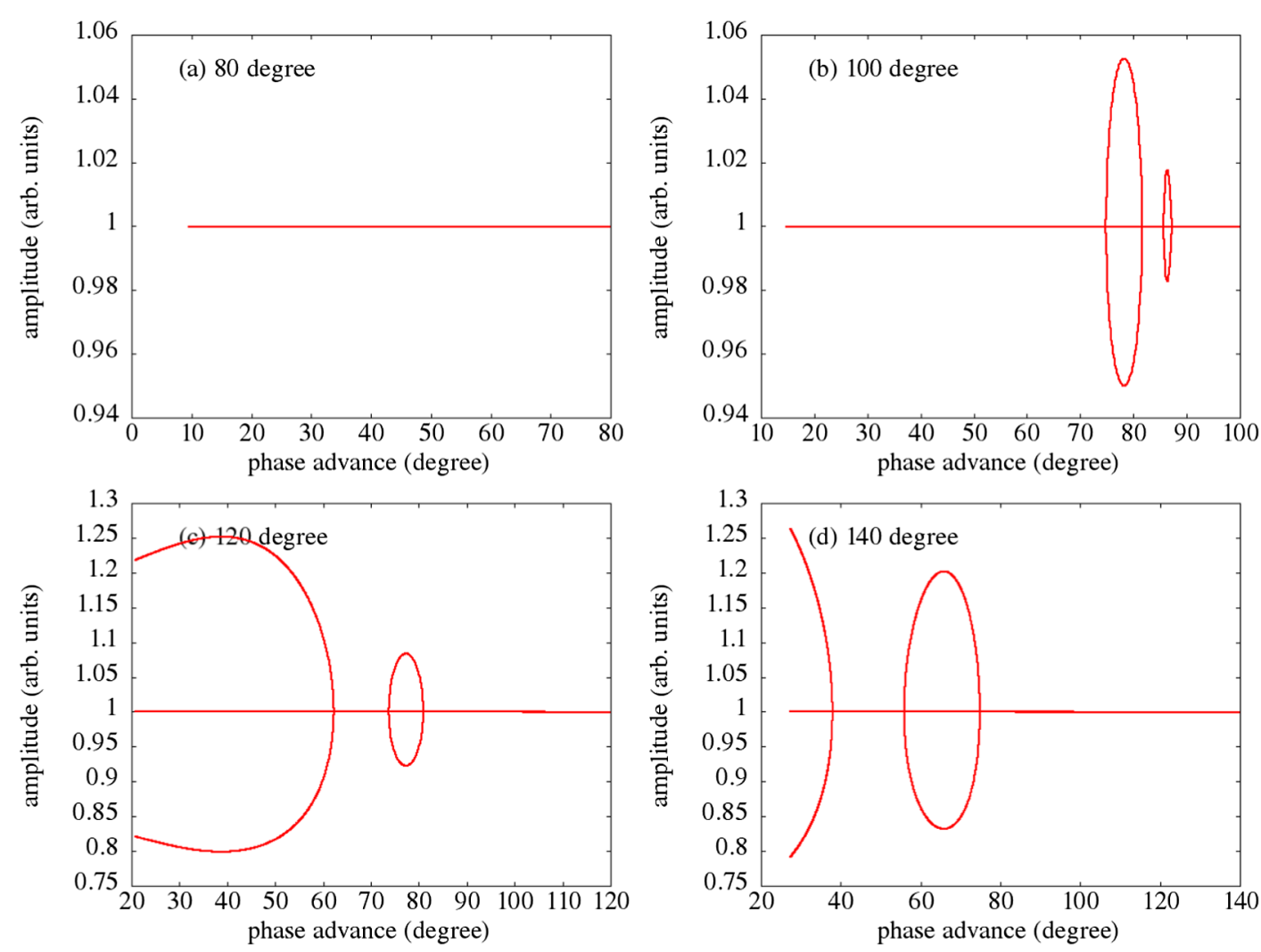

FIG. 4. The 2D envelope mode growth rate amplitudes as a function of depressed transverse phase advance for (a) $80^{\circ}$, (b) $100^{\circ}$, (c) $120^{\circ}$, and (d) $140^{\circ}$ zero current transverse phase advances in a periodic solenoid channel.

where $M_{6}(s)$ denotes the $6 \times 6$ transfer matrix solution of $\xi(s)$ and $M_{6}(0)$ is a $6 \times 6$ unit matrix. The above ordinary differential equation can be solved using the matched envelope solutions and numerical integration. Similar to the $2 \mathrm{D}$ envelope instability model, the stability of these envelope perturbations is determined by the eigenvalues of the transfer matrix $M_{6}(L)$ through one lattice period. For the envelope oscillation to be stable, all six eigenvalues (three pairs) of the $M_{6}(L)$ have to stay on the unit circle. The amplitude of the eigenvalue gives the envelope mode growth (or damping) rate through one lattice period, while the phase of the eigenvalue yields the mode oscillation frequency. When the amplitude of any eigenvalue is greater than one, the envelope oscillation becomes unstable.

\section{ENVELOPE INSTABILITY IN A PERIODIC SOLENOID AND RF CHANNEL}

I first studied the envelope instability in a transverse solenoid focusing and longitudinal rf focusing periodic channel. A schematic plot of this periodic channel is shown in Fig. 1. Each period of the channel consists of a $0.2 \mathrm{~m}$ solenoid and a $0.1 \mathrm{~m}$ rf bunching cavity. The total length of the period is $0.5 \mathrm{~m}$. The proton bunch has a kinetic energy of $150 \mathrm{MeV}$ and normalized rms emittances of $0.2 \mu \mathrm{m}, 0.2 \mu \mathrm{m}$, and $0.2 \mu \mathrm{m}$ in horizontal, vertical, and longitudinal directions respectively. Figures 2 and 3 show the 3D envelope mode growth rate amplitudes $(|\lambda|)$ and phases $(\phi)$ as a function of transverse depressed phase advance for different zero current transverse and longitudinal phase advances. As a comparison, we also show in Figs. 4 and 5 the 2D envelope mode growth rate amplitudes and phases as a function of depressed transverse phase advance for the same zero current transverse phase advances. Here, the 2D periodic solenoid channel has the same length of period as the $3 \mathrm{D}$ channel. It is seen that in the 2D periodic solenoid channel, the envelope instability occurs when the zero current phase advance is over $90^{\circ}$. In the $3 \mathrm{D}$ periodic solenoid-rf channel, the envelope instability occurs even with the zero current transverse phase advance $80^{\circ}$ but longitudinal phase advance beyond $90^{\circ}$ as shown in Fig. 2(a2). There is no instability if both the transverse zero current phase advance and the longitudinal zero current phase advance are below $90^{\circ}$ as seen in Fig. 2(a1). For the 3D envelope modes, when the longitudinal zero current phase advance below $90^{\circ}$ and the transverse zero current phase above $90^{\circ}$ as shown in Figs 2(b1), 2(c1), and 2(d1), the instability stopband becomes broader as the zero current longitudinal phase advance increases. This is probably because the longitudinal synchrotron motion helps bring particles with different depressed transverse tunes into the resonance. A faster synchrotron motion results in more particles falling into the resonance and hence a broader instability stopband. For small longitudinal zero current 

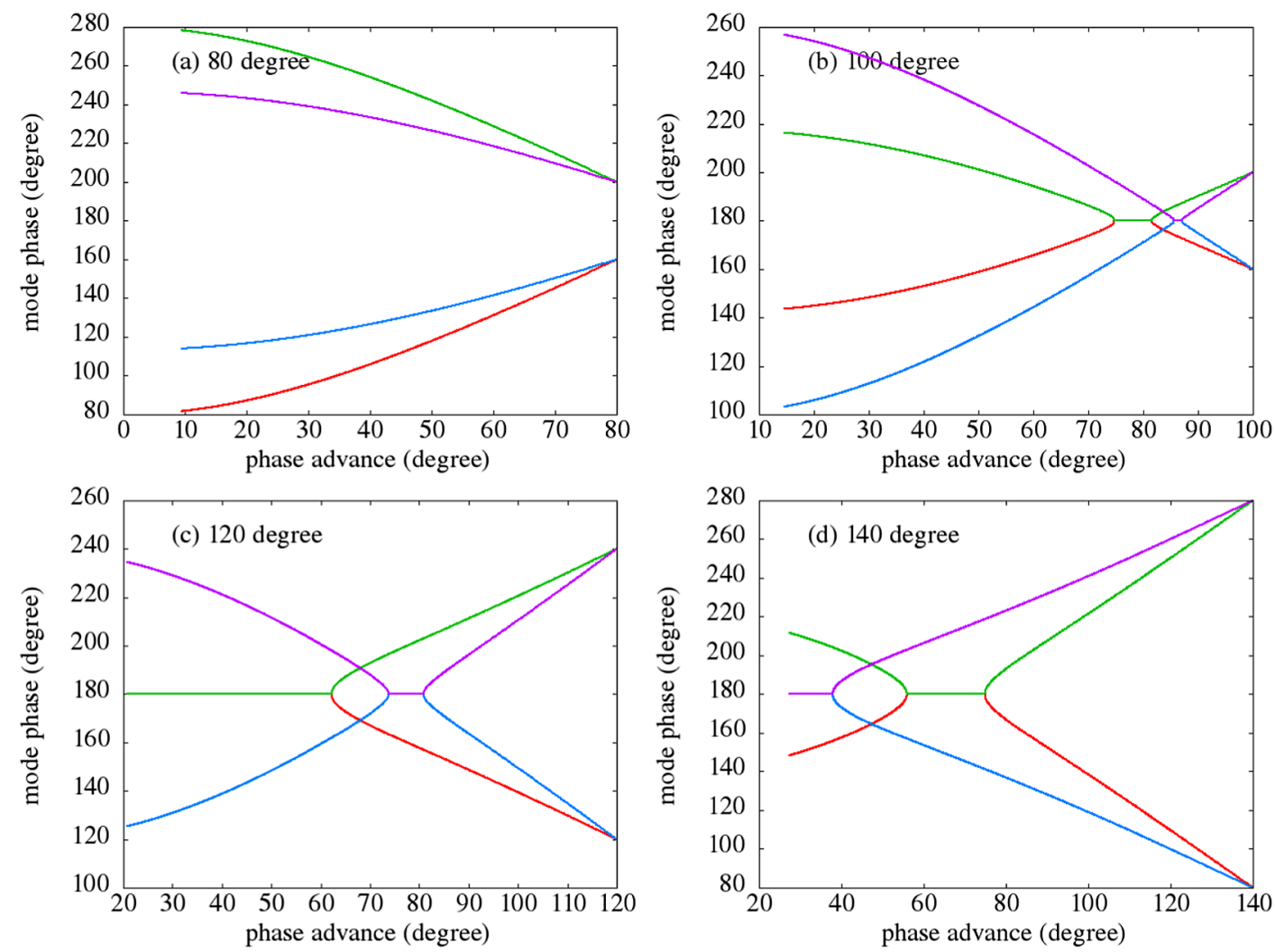

FIG. 5. The 2D envelope mode phases as a function of depressed transverse phase advance for (a) $80^{\circ}$, (b) $100^{\circ}$, (c) $120^{\circ}$, and (d) $140^{\circ}$ zero current transverse phase advances in a periodic solenoid channel. The four colors are the phases of the four eigenvalues.
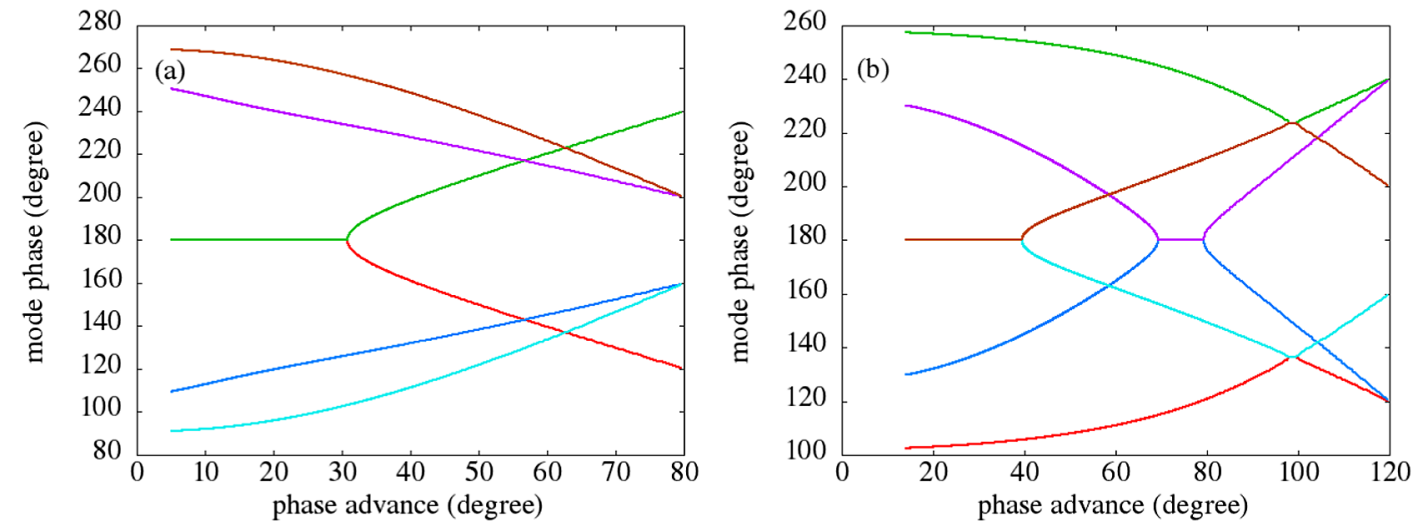

FIG. 6. The 3D envelope mode phases as a function of the depressed transverse phase advance for zero current (a) transverse $80^{\circ}$ and longitudinal $120^{\circ}$, (b) transverse $120^{\circ}$ and longitudinal $80^{\circ}$ phase advance in a periodic solenoid channel. The six colors are the phases of the six eigenvalues.

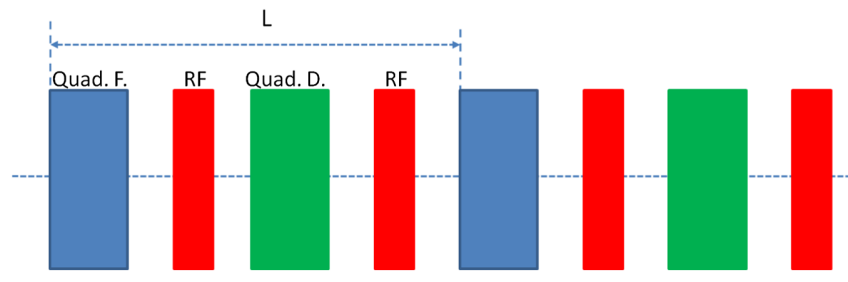

FIG. 7. Schematic plot of a periodic quadrupole and $\mathrm{rf}$ channel. phase advance (e.g., $20^{\circ}$ ), the $3 \mathrm{D}$ envelope mode show the stopband similar to that of the 2D envelope mode. When the longitudinal zero current phase advance is above $90^{\circ}$, as shown in Figs. 2(b2), 2(c2), and 2(d2), the 3D envelope instability shows more complicated structure and larger instability stopband width than the 2D envelope instability.

In the 2D periodic transverse solenoid focusing channel, for a coasting beam with equal horizontal and vertical 

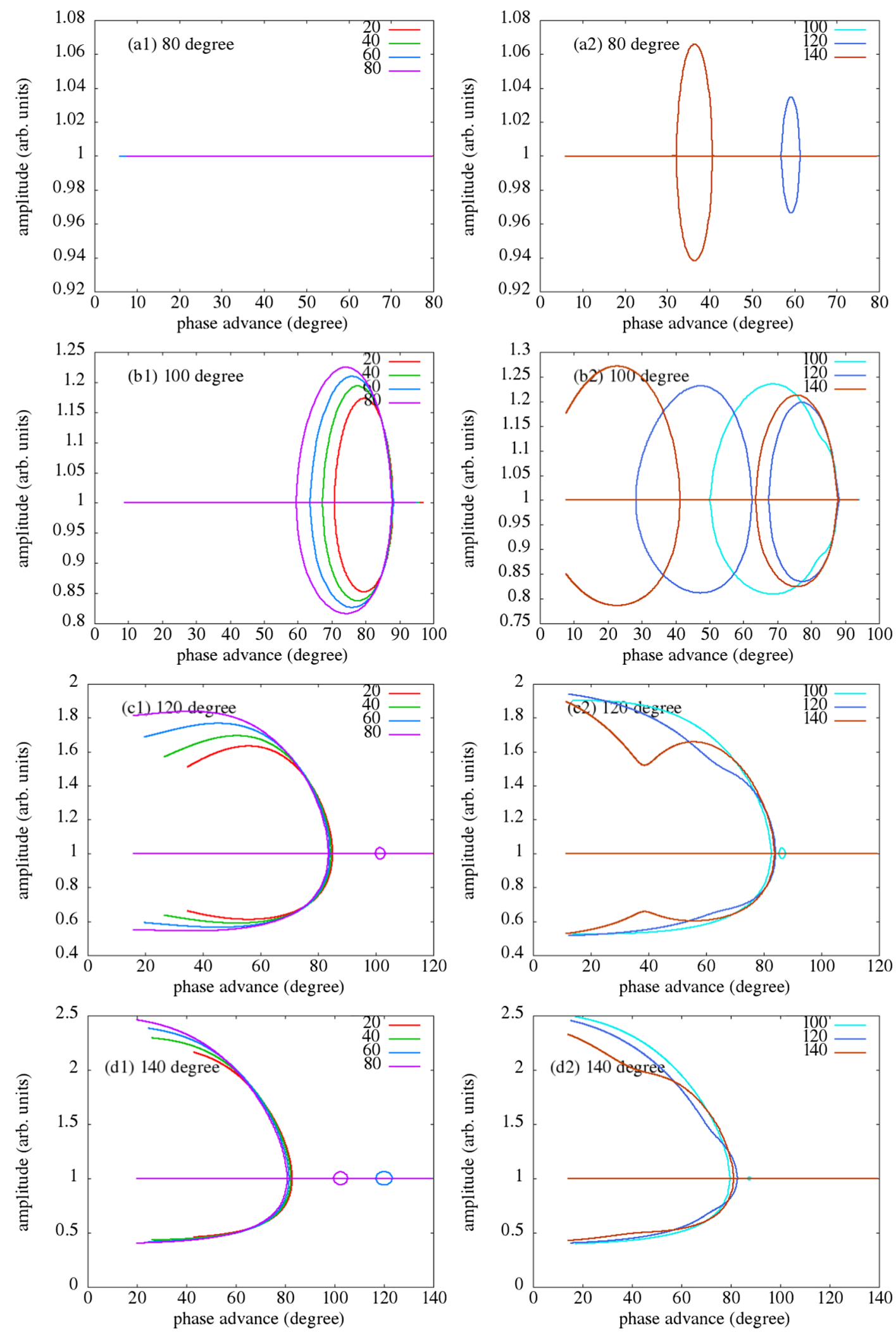

FIG. 8. The 3D envelope mode growth rate amplitudes as a function of depressed transverse phase advance with $20^{\circ}, 40^{\circ}, 60^{\circ}, 80^{\circ}$, $100^{\circ}, 120^{\circ}$, and $140^{\circ}$ zero current longitudinal phase advances for (a) $80^{\circ}$, (b) $100^{\circ}$, (c) $120^{\circ}$, and (d) $140^{\circ}$ zero current transverse phase advances in a periodic quadrupole-rf channel. 

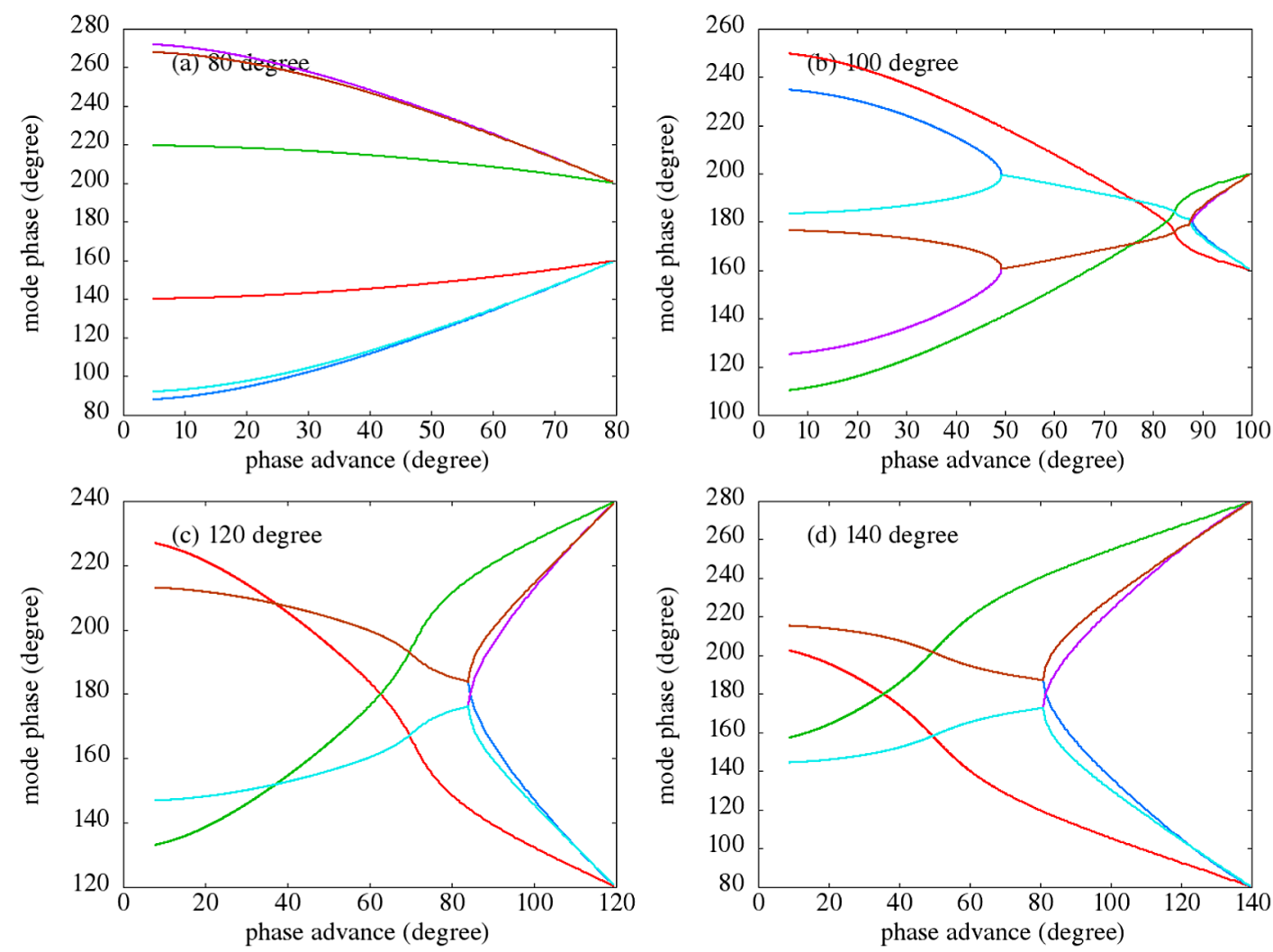

FIG. 9. The 3D envelope mode phases as a function of depressed transverse phase advance with (a) $80^{\circ}$, (b) $100^{\circ}$, (c) $120^{\circ}$, and (d) $140^{\circ}$ zero current longitudinal and transverse phase advances in a periodic quadrupole-rf channel. The six colors are the phases of the six eigenvalues.

emittances, it is seen in Fig. 5, the envelope instabilities are due to the $180^{\circ}$ half-integer parametric resonance. However, for a bunched beam, as shown in Fig. 3, besides the $180^{\circ}$ half-integer resonance, there are also confluent resonances where two envelope modes have the same frequencies and resonate with each other. The existence of both instability mechanisms results in more complicated structure as shown in Figs. 2(b2), 2(c2), and 2(d2).

The 3D envelope instability shows asymmetry between the transverse direction and the longitudinal direction in the 3D periodic solenoid and rf channel. Figure 6 shows the envelope mode phases as a function of depressed transverse phase advance for a case with zero current $80^{\circ}$ transverse phase advance and $120^{\circ}$ longitudinal phase advance, and a case with zero current $120^{\circ}$ transverse phase advance and $80^{\circ}$ longitudinal phase advance. The envelope mode growth rate amplitudes for both cases are shown in Figs. 2(a2) and 2(c1). For the $80^{\circ}$ zero current transverse phase advance, there is only one major unstable stopband below $30^{\circ}$ depressed transverse phase advance due to half-integer parametric resonance as shown in the left plot of Fig. 6 . For the $120^{\circ}$ zero current transverse phase advance, there are three unstable regions, two due to the half-integer parameter resonance and one due to the confluent resonance as shown in the right plot of Fig. 6. This asymmetry is related to the two degrees of freedom in the transverse plane while only one in the longitudinal direction.

\section{ENVELOPE INSTABILITY IN A PERIODIC QUADRUPOLE-RF CHANNEL}

Next, we studied the 3D envelope instability in a periodic transverse quadrupole focusing and longitudinal rf focusing channel for the same bunched proton beam. A schematic plot of this periodic channel is shown in Fig. 7. Each peroid of the channel consists of a $0.2 \mathrm{~m}$ focusing quadrupole, a $0.1 \mathrm{~m}$ rf focusing cavity, a $0.2 \mathrm{~m}$ defocusing quadrupole, and another $0.1 \mathrm{~m} \mathrm{rf}$ bunching cavity. The total length of the period is $1.0 \mathrm{~m}$. Figures 8 and 9 show the 3D envelope mode growth rate amplitudes and phases as a function of transverse depressed phase advance for different zero current transverse and longitudinal phase advances. As a comparison, we also show in Figs 10 and 11 the 2D envelope mode growth rate amplitudes and phases as a function of the depressed phase advance for different zero current phase advances. Here, the 2D periodic quadrupole channel has the same length of period as the $3 \mathrm{D}$ channel. It is seen that in the 2D periodic quadrupole channel, the envelope instability occurs when the zero current phase advance is over $90^{\circ}$. There is no instability when the zero current phase advance is below $90^{\circ}$. In the $3 \mathrm{D}$ 

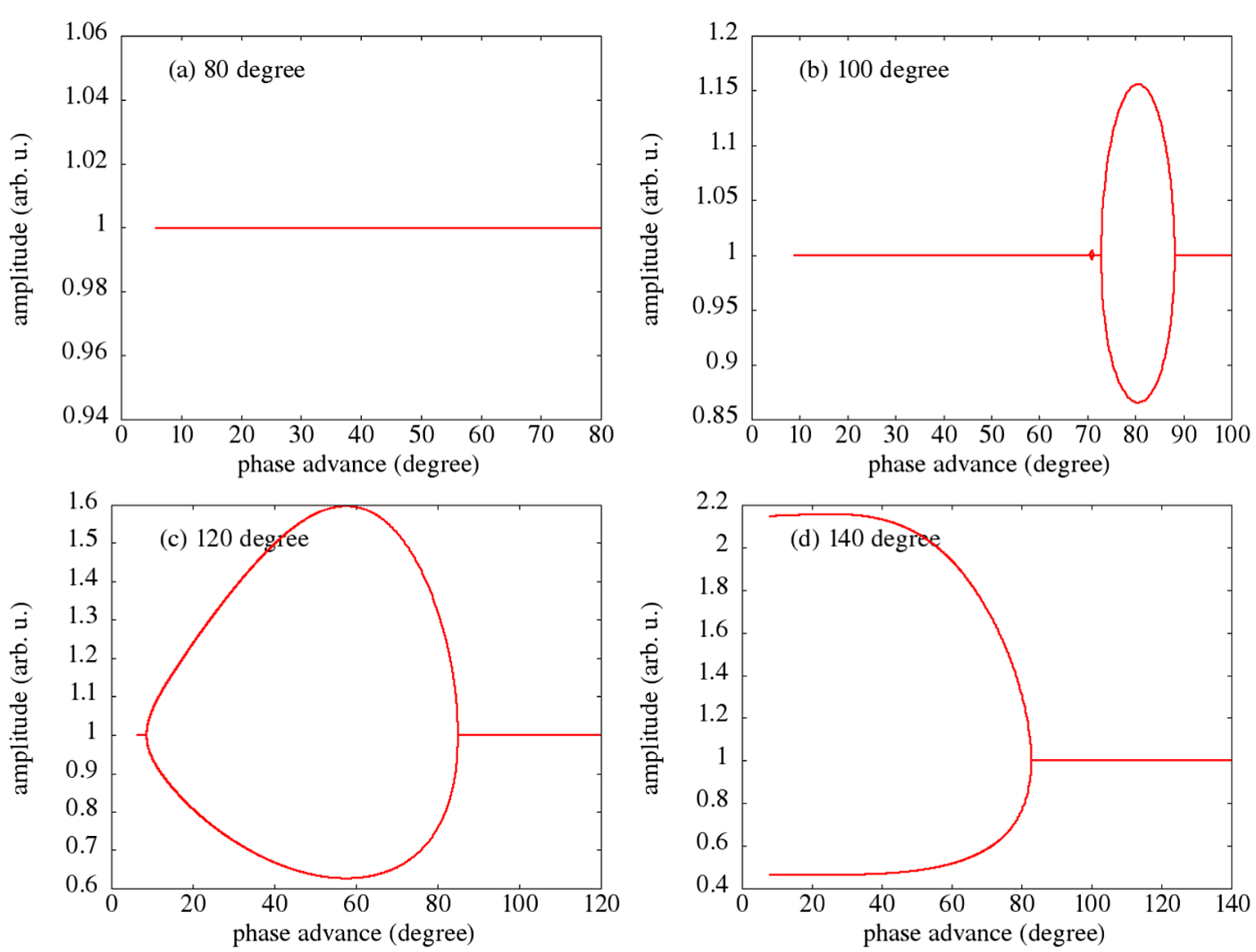

FIG. 10. The 2D envelope mode growth rate amplitudes as a function of depressed transverse phase advance for (a) $80^{\circ}$, (b) $100^{\circ}$, (c) $120^{\circ}$, and (d) $140^{\circ}$ zero current transverse phase advances in a periodic quadrupole channel.

periodic quadrupole-rf channel, the envelope instability occurs even with the zero current transverse phase advance $80^{\circ}$ but the longitudinal phase advance beyond $100^{\circ}$ in Fig. 8(a2). There is no instability if both the transverse zero current phase advance and the longitudinal zero current phase advance are below $90^{\circ}$. For the 3D envelope modes, when the longitudinal zero current phase advance is below $90^{\circ}$ and the transverse zero current phase above $90^{\circ}$ as shown in Figs. 8(b1), 8(c1), and 8(d1), the instability stopband width increases with the increase of the zero current longitudinal phase advance. For small longitudinal zero current phase advance (e.g., $20^{\circ}$ ), the $3 \mathrm{D}$ envelope modes instability stopband is similar to that of the 2D envelope modes. For the $100^{\circ}$ zero current transverse phase advance case, when the zero current longitudinal phase advance is beyond $90^{\circ}$, the stopband becomes more complicated and shows multiple stopbands. For the transverse zero current $120^{\circ}$ and $140^{\circ}$ phase advances, the instability stopbands do not change significantly with the increase of zero current longitudinal phase advance. This is due to the fact that when the transverse zero current phase advance is beyond $100^{\circ}$, most parameter space (transverse depressed tune) below $90^{\circ}$ becomes unstable caused by the confluent resonance. Further increasing the zero current longitudinal phase advance beyond $90^{\circ}$ will not enlarge that stopband any more.
In the periodic transverse quadrupole focusing channel, it is seen in Fig. 11, the 2D envelope instabilities are mainly due to the confluent resonance between the two envelope modes when their phases become equal. This appears still to be valid in the $3 \mathrm{D}$ periodic quadrupole-rf channel as shown in Fig. 9.

The 3D envelope instability shows asymmetry between the transverse and the longitudinal direction in the 3D periodic quadrupole and rf channel too. Figure 12 shows the envelope mode phases as a function of depressed transverse phase advance for a case with zero current $80^{\circ}$ transverse phase advance and $120^{\circ}$ longitudinal phase advance, and a case with zero current $120^{\circ}$ transverse phase advance and $80^{\circ}$ longitudinal phase advance. The envelope mode amplitudes are shown in Figs. 8(a2) and 8(c1) for this comparison. For the $80^{\circ}$ zero current transverse phase advance, there is only one major unstable region around $60^{\circ}$ depressed transverse phase advance due to the confluent resonance. For the $120^{\circ}$ zero current phase advance, there are two unstable regions due to two confluent resonances.

In the above periodic quadrupole and rf channel, we assumed that the two rf cavities have the same longitudinal focusing strength. The longitudinal focusing period is half of the transverse focusing period. This accounts for the absence of the envelope instability for the zero current $80^{\circ}$ 

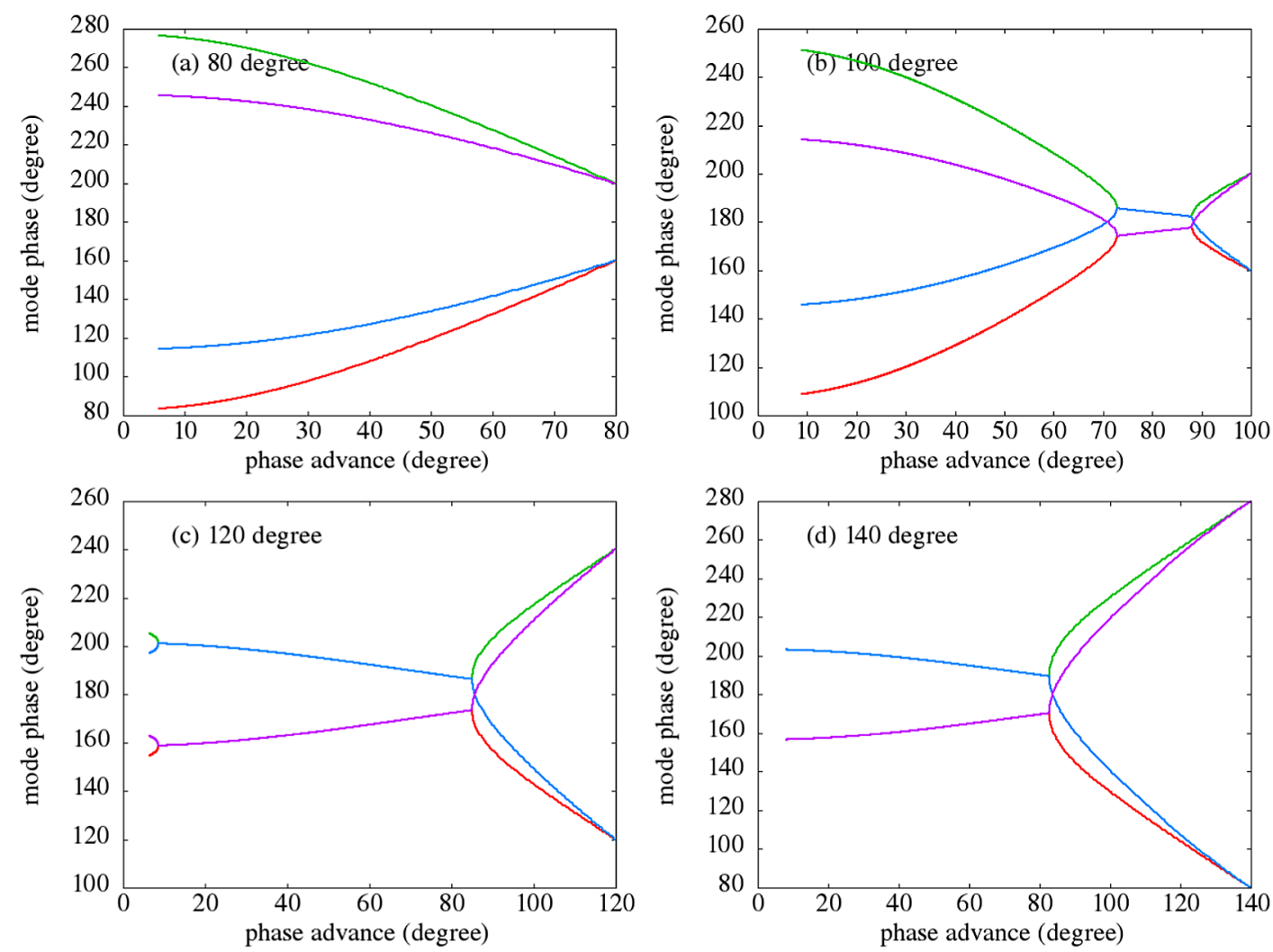

FIG. 11. The 2D envelope mode phases as a function of depressed transverse phase advance for (a) $80^{\circ}$, (b) $100^{\circ}$, (c) $120^{\circ}$, and (d) $140^{\circ}$ zero current transverse phase advances in a periodic quadrupole channel. The four colors are the phases of the four eigenvalues.
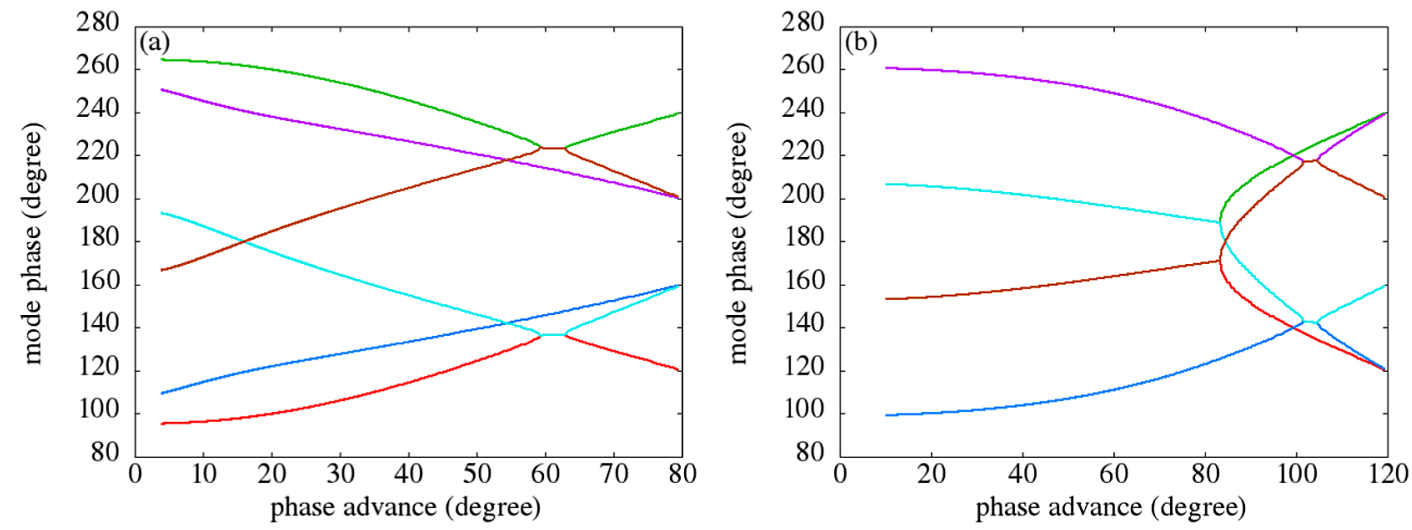

FIG. 12. The 3D envelope mode phases as a function of depressed transverse phase advance for zero current (a) transverse $80^{\circ}$ and longitudinal $120^{\circ}$, (b) transverse $120^{\circ}$ and longitudinal $80^{\circ}$ phase advance in a periodic quadrupole channel. The six colors are the phases of the six eigenvalues.

transverse phase advance and $100^{\circ}$ longitudinal phase in the periodic quadrupole and $\mathrm{rf}$ channel. The envelope instability stopband is observed in the periodic solenoid and rf channel with the same zero current phase advances as shown in Fig. 2(a2). The absence of instability for longitudinal zero current phase advance $100^{\circ}$ was also observed in 3D macroparticle simulations in Ref. [22]. Now, we break the symmetry of two rf longitudinal focusing cavities, the longitudinal focusing period becomes the same as the transverse focusing period. The envelope instability occurs for these zero current phase advances in a periodic quadrupole and if channel. Figure 13 show the envelope mode growth rate amplitudes and phases as a function of transverse depressed phase advances with about $10 \%, 20 \%$, and $30 \%$ deviation from the original setting of the two rf cavities (one cavity plus that percentage and the other one 

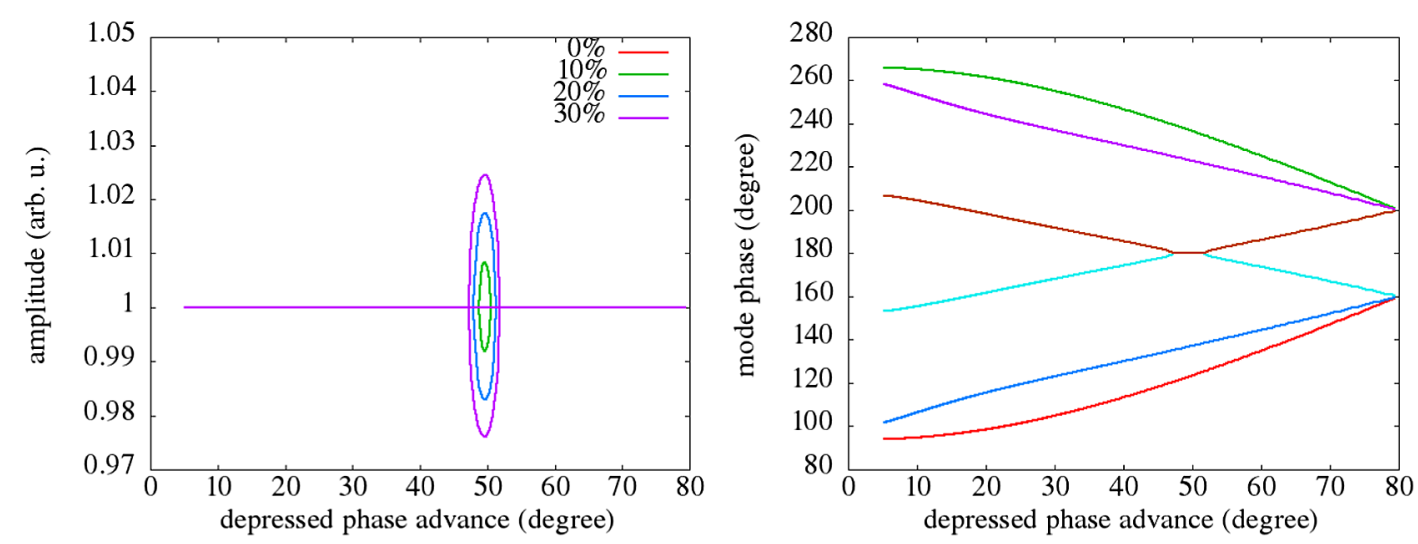

FIG. 13. The 3D envelope mode (left) growth rate amplitudes and (right) phases as a function of the transverse depressed phase advance with $10 \%, 20 \%$, and $30 \%$ deviations from the original rf cavity setting in a periodic quadrupole-rf channel. The six colors in the right plot are the phases of the six eigenvalues.
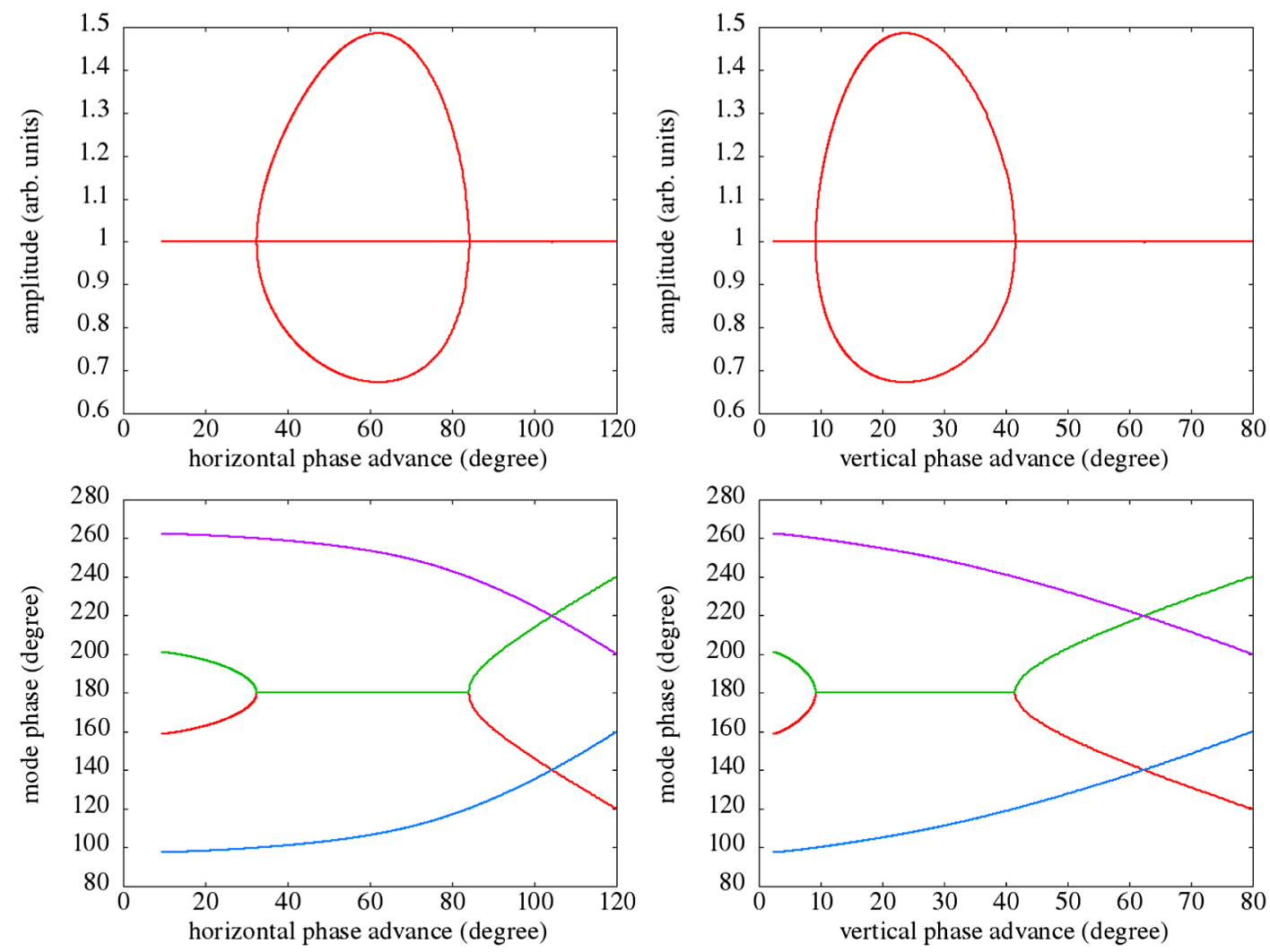

FIG. 14. The 2D envelope mode (top) growth rate amplitudes and (bottom) phases as a function of depressed phase advance with asymmetric zero current phase advances $\left(80^{\circ}\right.$ in one direction and $120^{\circ}$ in another direction) in a periodic quadrupole channel. The four colors in the bottom plots are the phases of the four eigenvalues.

minus that percentage). It is seen that as the asymmetry between the two rf cavity increases, the instability stopband width also increases. Before breaking of the symmetry of two rf cavities, the longitudinal phase advance per longitudinal period is $50^{\circ}$. After the breaking of the symmetry, the longitudinal period becomes the same as the lattice period and the phase advance becomes $100^{\circ}$. Such a zero current phase advance results in half integer parametric resonance as shown in Fig. 13.

In above 3D periodic solenoid/quadrupole and rf transport channels, I have assumed that in transverse plane, the zero current phase advances in horizontal direction and the vertical direction are the same. Furthermore, the bunch has the same emittances in both horizontal and vertical 

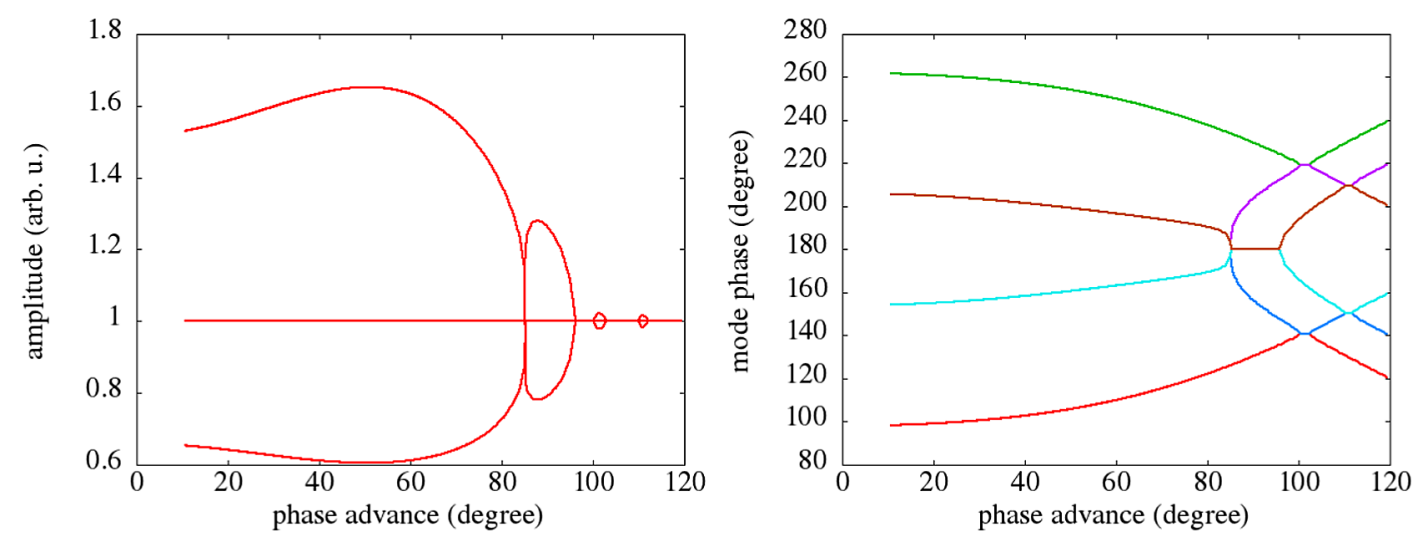

FIG. 15. The 3D envelope mode (left) growth rate amplitudes and (right) phases as a function of the horizontal depressed phase advances with zero current phase advances $120^{\circ}$ in horizontal, $110^{\circ}$ in vertical, and $80^{\circ}$ in longitudinal direction in a periodic quadrupole-rf channel. The six colors in the right plot are the phases of the six eigenvalues.

directions. This implies a two-dimensional transverse and longitudinal periodic system (i.e. $r-z$ ). As a comparison, we also calculated the envelope mode growth rate amplitudes and phases for a true two-dimensional periodic quadrupole channel with different zero current phase advances in the horizontal and the vertical direction $\left(120^{\circ}\right.$ in the horizontal direction and $80^{\circ}$ in the vertical direction). Figure 14 shows the $2 \mathrm{D}$ envelope mode growth rate amplitudes and phases as a function of the depressed horizontal and vertical phase advance. Comparing the 2D envelope mode growth rate amplitudes and phases in above plot with those of the 3D envelope mode with the same zero current phase advances in Figs. 2(a2) and 8(a2) $\left(80^{\circ}\right.$ in transverse and $120^{\circ}$ in longitudinal) and Figs. 2(c1) and $8(\mathrm{c} 1)$ $\left(120^{\circ}\right.$ in transverse and $80^{\circ}$ in longitudinal), it is seen that the 2D envelope instability shows somewhat similar structure to the $3 \mathrm{D}$ envelope instability in a periodic solenoid-rf channel with transverse zero current phase advance $80^{\circ}$ and longitudinal phase advance $120^{\circ}$. The major instabilities in both cases are caused by the half-integer parametric resonance. The $3 \mathrm{D}$ envelope modes in a periodic quadrupole-rf channel shows quite different instability stopband from the $2 \mathrm{D}$ envelope modes. Also the $3 \mathrm{D}$ envelope instability in quadrupole channel is caused by the confluent resonance while the $2 \mathrm{D}$ asymmetric envelope instability in the quadrupole channel is mainly caused by the half-integer parametric resonance.

I also explored 3D envelope instabilities with nonequal transverse zero current phase advances in the horizontal direction and the vertical direction. Figure 15 shows the 3D envelope mode amplitudes and phases as a function of the depressed horizontal tune with zero current phase advance $120^{\circ}$ in the horizontal direction, $110^{\circ}$ in the vertical direction, and $80^{\circ}$ in the longitudinal direction in the periodic quadrupole and rf channel. Comparing the above figure with the zero current $120^{\circ}$ transverse phase advance and $80^{\circ}$ longitudinal phase advance case in Fig. 8(c1), it is seen that 3D instability stopband from the nonequal transverse focusing becomes broader. Instead of one major instability stopband and a minor stopband in the equal transverse phase advance case, now there are four stopbands (two major stopbands and two minor stopbands) for the transverse $120^{\circ}$ and $110^{\circ}$ phase advances. Besides the confluent resonance, there also appears a half-integer parametric resonance when the transverse symmetry is broken. Breaking the transverse symmetry results in more resonances of these envelope modes. This suggests that keeping the same zero current phase advance in both the horizontal and the vertical directions might help reduce the parameter region of the envelope instability.

\section{CONCLUSIONS}

In this paper, we studied the envelope instability for a bunched beam using a set of three-dimensional envelope equations in a periodic solenoid and rf focusing channel and a periodic quadrupole and rf focusing channel. This study showed that when the transverse zero current phase advance is below $90^{\circ}$, the beam envelope can still become unstable if the longitudinal zero current phase advance is beyond $90^{\circ}$. For the transverse zero current phase advance beyond $90^{\circ}$, the instability stopband becomes broader with the increase of longitudinal focusing strength and even shows different structure from the $2 \mathrm{D}$ case when the longitudinal zero current phase advance is beyond $90^{\circ}$.

The 3D envelope instability shows asymmetry between the longitudinal focusing and the transverse focusing. The instability shows broader stopband when the transverse zero current phase advance is beyond $90^{\circ}$ than that when the longitudinal zero current phase advance is beyond $90^{\circ}$. In the $3 \mathrm{D}$ periodic quadrupole and $\mathrm{rf}$ channel, for the transverse zero current phase advance $80^{\circ}$, the envelope modes stay stable for the longitudinal $100^{\circ}$ zero current phase advance due to the symmetry of two longitudinal focusing rf cavities. Breaking the symmetry of two cavities 
results in the envelope instability with a finite stopband. Breaking the horizontal and vertical focusing symmetry in the transverse plane also increases the envelope instability stopband width. This suggests that a more symmetric accelerator lattice design might help reduce the parameter space of the envelope instability.

In this study, we used a transverse solenoid, longitudinal rf focusing lattice and a transverse quadrupole, longitudinal rf focusing lattice to illustrate the 3D envelope instability of a bunched beam in periodic transport channels. This model can be readily applied to the study of stability of bunched beam transporting in other periodic focusing lattice, e.g., a FOFO-DODO lattice. The above results will still be valid qualitatively even though the exact width of instability stopband can be different. This is because the space-charge driven envelope instability depends more on the zero-current phase advance per period and the depressed phase advance per period with current than the detailed layout of focusing elements. The dependence of the instability stopband on accelerator lattice parameters is not analytically available and can be explored numerically in future study.

\section{ACKNOWLEDGMENTS}

Work supported by the U.S. Department of Energy under Contract No. DE-AC02-05CH11231. I would like to thank Dr. R. D. Ryne for the use of his $2 \mathrm{D}$ and $3 \mathrm{D}$ envelope codes. This research used computer resources at the National Energy Research Scientific Computing Center.

[1] I. Hofmann, L. J. Laslett, L. Smith, and I. Haber, Stability of the Kapchinskij-Vladimirskij (K-V) distribution in long periodic transport systems, Part. Accel. 13, 145 (1983).

[2] J. Struckmeier and M. Reiser, Theoretical studies of envelope oscillations and its instabilities of mismatched intense charged-particle beams in periodic focusing channels, Part. Accel. 14, 227 (1984).

[3] I. Hofmann, Stability of anisotropic beams with space charge, Phys. Rev. E 57, 4713 (1998).

[4] R. C. Davidson, H. Qin, and G. Shvets, A Paul trap configuration to simulate intense non-neutral beam propagation over large distances through a periodic focusing quadrupole magnetic field, Phys. Plasmas 7, 1020 (2000).

[5] H. Okamoto and K. Yokoya, Parametric resonances in intense one-dimensional beams propagating through a periodic focusing channel, Nucl. Instrum. Methods Phys. Res., Sect. A 482, 51 (2002).

[6] A. V. Fedotov and I. Hofmann, Half-integer resonance crossing in high-intensity rings, Phys. Rev. ST Accel. Beams 5, 024202 (2002).

[7] A. V. Fedotov, I. Hofmann, R. L. Gluckstern, and H. Okamoto, Parametric collective resonances and spacecharge limit in high-intensity rings, Phys. Rev. ST Accel. Beams 6, 094201 (2003).
[8] S. M. Lund and B. Bukh, Stability properties of the transverse envelope equations describing intense ion beam transport, Phys. Rev. ST Accel. Beams 7, 024801 (2004).

[9] M. Tiefenback, Ph.D. thesis, University of California at Berkeley [Lawrence Berkeley National Laboratory Report No. LBL-22465, 1986].

[10] E. P. Gilson, M. Chung, R. C. Davidson, P. C. Efthimion, and R. Majeski, Transverse beam compression on the Paul trap simulator experiment, Phys. Rev. ST Accel. Beams 10, 124201 (2007).

[11] L. Groening, W. Barth, W. Bayer, G. Clemente, L. Dahl, P. Forck, P. Gerhard, I. Hofmann, M. S. Kaiser, M. Maier, S. Mickat, and T. Milosic, Experimental Evidence of the $90^{\circ}$ Stop Band in the GSI UNILAC, Phys. Rev. Lett. 102, 234801 (2009).

[12] D. Jeon, L. Groening, and G. Franchetti, Fourth order resonance of a high intensity linear accelerator, Phys. Rev. ST Accel. Beams 12, 054204 (2009).

[13] K. Fukushima, K. Itoa, H. Okamoto, S. Yamaguchi, K. Moriya, H. Higaki, T. Okano, and S. M. Lund, Experimental verification of resonance instability bands in quadrupole doublet focusing channels, Nucl. Instrum. Methods Phys. Res., Sect. A 733, 18 (2014).

[14] C. Li and Y. L. Zhao, Envelope instability and the fourth order resonance, Phys. Rev. ST Accel. Beams 17, 124202 (2014).

[15] C. Li and Q. Qin, Space charge induced beam instability in periodic focusing channel, Phys. Plasmas 22, 023108 (2015).

[16] I. Hofmann and O. Boine-Frankenheim, Space-Charge Structural Instabilities and Resonances in High-Intensity Beams, Phys. Rev. Lett. 115, 204802 (2015).

[17] D. Jeon, J. H. Jang, and H. Jin, Interplay of space-charge fourth order resonance and envelope instability, Nucl. Instrum. Methods Phys. Res., Sect. A 832, 43 (2016).

[18] O. Boine-Frankenheim, I. Hofmann, and J. Struckmeier, Parametric sum envelope instability of periodically focused intense beams, Phys. Plasmas 23, 090705 (2016).

[19] I. Hofmann and O. Boine-Frankenheim, Parametric instabilities in 3D periodically focused beams with space charge, Phys. Rev. Accel. Beams 20, 014202 (2017).

[20] K. Ito, H. Okamoto, Y. Tokashiki, and K. Fukushima, Coherent resonance stop bands in alternating gradient beam transport, Phys. Rev. Accel. Beams 20, 064201 (2017).

[21] Y. Yuan, O. Boine-Frankenheim, and I. Hofmann, Modeling of second order space charge driven coherent sum and difference instabilities, Phys. Rev. Accel. Beams 20, 104201 (2017).

[22] I. Hofmann and O. Boine-Frankenheim, Revisiting the Longitudinal $90^{\circ}$ Limit in High Intensity Linear Accelerators, Phys. Rev. Lett. 118, 114803 (2017).

[23] I. Hofmann, Space Charge Physics for Particle Accelerators (Springer, New York, 2017).

[24] I. Kapchinskiy and V. Vladimirskiy, in 2nd Conference on High Energy Accelerators (CERN, Geneva, 1959), p. 274.

[25] R. Ryne, Los Alamos Report No. LA-UR-95-391; http:// xxx.lanl.gov/abs/acc-phys/9502001.

[26] K. Bongardt, M. Pabst, and A. Letchford, in Proceedings of the 19th International Linear Accelerators Conference, Chicago, IL, 1998 (NTIS, Springfield, VA, 1998), p. 824.

[27] C. Allen, in Proceedings of the 2nd ICFA Advanced Accelerator Workshop, edited by J. Rosenzweig 
and L. Serafini (World Scientific, Singapore, 2000), p. 173.

[28] J. Qiang and R. D. Ryne, Beam halo studies using a threedimensional particle-core model, Phys. Rev. ST Accel. Beams 3, 064201 (2000).

[29] M. Comunian, A. Pisent, A. Bazzani, G. Turchetti, and S. Rambaldi, Frequency map analysis of a three-dimensional particle in the core model of a high intensity linac, Phys. Rev. ST Accel. Beams 4, 124201 (2001).
[30] F. J. Sacherer, RMS envelope equations with space charge, IEEE Trans. Nucl. Sci. 18, 1105 (1971).

[31] Thomas P. Wangler, RF Linear Accelerators, 2nd ed. (Wiley, New York, 2008).

[32] G. Fubiani, J. Qiang, E. Esarey, W. P. Leemans, and G. Dugan, Space charge modeling of dense electron beams with large energy spreads, Phys. Rev. ST Accel. Beams 9, 064402 (2006). 